\title{
Sécrétions apocrines et glandes annexes
}

\author{
Robert SULLIVAN, Gilles FRENETTE, Christine LEGARE \\ Centre de Recherche en Biologie de la Reproduction et Département d'Obstétrique-Gynécologie, Faculté de \\ Médecine, Université Laval, Québec
}

\section{RESUME}

Le spermatozoïde quittant le testicule est incapable d'activité transcriptionnelle ou traductionnelle. Malgré cela, la composition en macromolécules de la surface du spermatozoïde subit des modifications majeures au cours de son passage dans le tractus reproducteur mâle. Ceci s'explique par l'interaction entre les sécrétions des différentes glandes du tractus reproducteur mâle et le spermatozoïde. Le mécanisme d'ancrage à la surface du spermatozoïde de certaines protéines nouvellement acquises, particulièrement l'ancrage glycosylphosphatidyl inositol, suggère un mode de sécrétion apocrine le long du tractus reproducteur mâle. Ce type de sécrétion a été démontré au niveau de la prostate, du canal déférent et de l'épididyme.

Notre laboratoire s'est penché sur ce phénomène particulièrement au niveau épididymaire. Nous avons montré que l'épithélium épididymaire sécrète par voie apocrine des microvésicules membranaires nommées épididymosomes. Différentes protéines sont associées à ces vésicules riches en cholestérol et en sphingomyéline. Nous avons montré que certaines de ces protéines associées aux épididymosomes sont sélectivement transférées aux spermatozoïdes au cours du transit épididymaire.

En utilisant une approche protéomique, nous avons identifié certaines de ces protéines. Celles-ci comprennent les enzymes impliquées dans la voie métabolique des polyols. Nous avons également mis en évidence les interactions entre le MIF (Macrophage migration Inhibitory Factor) sécrété par l'épididyme et le spermatozoïde. Tant les polyols que le MIF peuvent être impliqués dans le contrôle de la motilité des spermatozoïdes au cours de leur transit épididymaire.

Ces résultats illustrent l'importance de la sécrétion apocrine dans la maturation des spermatozoïdes au cours de leur transit dans le tractus reproducteur mâle.

Mots clés : épididyme, exosomes, maturation, sécrétion, protéines

\section{INTRODUCTION}

Depuis plusieurs années, notre laboratoire s'intéresse aux modifications post-testiculaires impliquées dans l'acquisition du pouvoir fécondant du gamète mâle [49]. En utilisant le hamster comme modèle, nous avons identifié une protéine spermatique montrant une affinité spécifique d'espèce pour les glycoprotéines de la zone pellucide homologue [48]. Cette protéine nommée P26h, est localisée sur la membrane plasmique qui couvre l'acrosome du spermatozoïde de la queue épididymaire de hamster et est acquise au cours du transit épididymaire $[6,50]$. Des anticorps dirigés contre la P26h inhibent l'attachement du spermatozoïde mature à la zone pellucide lors d'essais in vitro et la P26h montre des propriétés immunocontraceptives lorsque utilisée pour immuniser activement des mâles hamsters $[7,26]$. L'ADNc codant pour la P26h a récemment été séquencé et la séquence en acides aminés déduite révèle que la $\mathrm{P} 26 \mathrm{~h}$ appartient à la famille des carbonyle réductases [25]. Des inhibiteurs spécifiques de la carbonyle réductase inhibent d'ailleurs la liaison du spermatozoïde à la zone pellucide in vitro [39]. Plusieurs faits expérimentaux appuient donc l'hypothèse que la P26h acquise par le spermatozoïde au cours de sa maturation dans l'épididyme est impliquée dans la liaison à la zone pellucide [47].

En utilisant les outils moléculaires développés lors de l'étude de la P26h du spermatozoïde de hamster, nous avons identifié des protéines orthologues chez d'autres espèces de mammifère en particulier chez l'humain et le taureau. Ces protéines ont été nommées $\mathrm{P} 34 \mathrm{H}$ et $\mathrm{P} 25 \mathrm{~b}$ respectivement $[8,9,41]$. Comme chez le hamster, la P34H humaine et la P25b bovine sont acquises au cours du transit épididymaire. Le transcrit de la $\mathrm{P} 34 \mathrm{H}$ en particu-

\section{Correspondance :}

Pr Robert SULLIVAN - Unité d'ontogénie-reproduction, Centre de recherche du CHUQ, Pavillon CHUL, 2705

Boul Laurier, Québec, Prov. Québec, Canada, G1V 4 G2 Email robert.sullivan@crchul.ulaval.ca 
lier est préférentiellement exprimé dans la région distale du corps épididymaire [35]. Chez le taureau comme chez l'humain, certains individus souffrant d'hypofertilité ou d'infertilité, produisent des spermatozoïdes caractérisés par l'absence ou la présence de faibles quantités de $\mathrm{P} 34 \mathrm{H}$ humaine ou de P25b bovine $[10,29,41]$. Nous avons montré que les spermatozoïdes provenant d'hommes infertiles et dépourvus de $\mathrm{P} 34 \mathrm{H}$ sont très peu performants dans des essais de liaison à la zone pellucide in vitro [10].

En utilisant le hamster, le bovin et I'humain, nous nous sommes intéressés au mode d'acquisition des protéines de la famille de la P26h au cours du transit épididymaire. L'analyse des séquences en acides aminés déduites à partir des séquences des ADNc codant pour la P34H humaine et la P26h de hamster révèle l'absence d'un peptide signal. Or, une protéine dépourvue de cette séquence en acides aminés n'est pas dirigée vers le Réticulum Endoplasmique au moment de sa synthèse et ne peut donc pas être sécrétée par la voie classique dite sécrétion mésocrine. Cette voie de sécrétion implique la fusion de granules de sécrétion provenant de l'appareil de Golgi avec la membrane plasmique de la cellule résultant en la libération par exocytose du contenu des granules de sécrétion dans le milieu extracellullaire [40]. Une autre particularité de ces protéines est leur type d'ancrage à la surface du spermatozoïde. Tel que nous l'avons démontré chez le taureau et le hamster, la P25b et la P26h sont ancrées à la membrane plasmique via un glycosyl phosphatidylinositol (GPI) et sont exposées au milieu extracellulaire [24, 34]. Ce type d'ancrage maintient une protéine à la membrane plasmique d'une cellule via deux chaînes d'acides gras. La protéine ainsi exposée vers le milieu extracellulaire est très solidement retenue à la membrane plasmique mais jouit d'une mobilité dans le plan de la membrane plasmique de beaucoup supérieure à celle des protéines membranaires intégrales. Classiquement, les protéines ancrées par un GPI à la surface de la membrane plasmique d'une cellule proviennent de la membrane des vésicules de sécrétion qui s'intègrent à la membrane plasmique lors de la sécrétion mésocrine. Un corollaire à ce dogme est qu'une protéine GPI ancrée à une cellule ne peut provenir du milieu extracellulaire. L'absence de peptide signal des protéines $\mathrm{P} 26 \mathrm{~h} / \mathrm{P} 34 \mathrm{H}$ synthétisées par les cellules principales et sécrétées dans la lumière épididymaire et leur ancrage GPI de la P26h à la surface du gamète mâle questionnent le mode de sécrétion utilisé par l'épididyme.

\section{LA SÉCRÉTION APOCRINE ET LE SYSTÈME REPRODUCTEUR MÂLE}

L'ancrage GPI et l'absence de peptide signal des protéines P26h excluent une sécrétion mésocrine et suggèrent une voie apocrine. Ce type de sécrétion dont l'exemple classique est la glande mammaire, implique la formation d'une excroissance cytoplasmique au niveau apical des cellules épithéliales. Cette excroissance qui contient un nombre restreint d'organites intracytoplasmiques se détache de la cellule et se désagrège dans le compartiment extracellulaire. Le contenu de ces excroissances est libéré et permet la dispersion dans le compartiment extracellulaire des protéi- nes cytoplasmiques qui n'ont pas de peptide signal et qui ne transitent donc pas par le Reticulum Endoplasmique, l'appareil de Golgi et les vésicules de sécrétion. Ces excroissances libèrent également de petites vésicules membranaires de diamètre variant entre 50 à $500 \mathrm{nM}$ nommées de façon générique "exosomes" [40]. Ce mécanisme de sécrétion a été particulièrement bien étudié au niveau du tractus reproducteur mâle $[5,30,46]$. Les prostasomes, des exosomes sécrétés par la prostate, ont en particulier suscité beaucoup d'intérêt. Décrits pour la première fois par Ronquist et al. [44], ces vésicules constituantes du liquide séminal ont fait l'objet de nombreuses études [45]. Une analyse protéomique récente a permis d'identifier pas moins de 139 protéines différentes associées aux prostasomes isolés du plasma séminal humain [52]. Différentes actitivités catalytiques sont associées aux prostasomes [33]. Plusieurs protéines associées aux prostasomes et aux exosomes de façon plus générale ont un ancrage GPI et sont dépourvus d'un peptide signal [32]. La présence de prostasomes dans le plasma séminal a depuis été démontrée chez plusieurs espèces incluant l'étalon $[2,38]$, le bélier [11] et le taureau [24]. Quelle que soit l'espèce étudiée, la composition des prostasomes est particulière. Le rapport molaire cholestérol/phospholipides de ces vésicules membranaires est très élevé, atteignant une valeur de 2 et la sphingomyéline compte pour près de $50 \%$ des phosholipides totaux [3]. Cette composition particulière suggère une organisation membranaire hautement ordonnée.

Les prostasomes présents dans le liquide séminal semblent jouer une multitude de fonctions. Ils possèderaient des propriétés immunosuppressives, antibactériennes et antioxydantes. Ils activeraient la coagulation sanguine et plusieurs activités enzymatiques leur seraient associées. Les prostasomes agiraient également sur les spermatozoïdes éjaculés en influençant les paramètres de motilité, en agissant au moment de la capacitation et en stimulant la réaction acrosomique $[4,45,46]$.

En plus des prostasomes, d'autres glandes annexes du système reproducteur mâle sécrètent des exosomes. Chez le taureau, les vésicules séminales sécrètent des exosomes qui se retrouvent dans le plasma séminal et qui s'apparentent donc aux prostasomes [1]. Chez le rat, la glande coagulante sécrète des exosomes auxquels sont associées des activités transglutaminase et carbonyle anhydrase impliquées dans la formation du bouchon vaginal $[5$, 28]. Le canal déférent chez la souris sécrète également des exosomes montrant une activité aldose réductase qui est transférée aux spermatozoïdes mais dont la fonction demeure indéterminée $[16,37]$. La sécrétion apocrine semble donc être utilisée par plusieurs organes le long du tractus reproducteur mâle. Bien que leur fonction demeure dans plusieurs cas à être déterminée, les exosomes joueraient des rôles prépondérants dans le processus global de la reproduction et plus particulièrement dans la protection et la modulation des fonctions spermatiques. Des exosomes sont également présents dans le système reproducteur femelle, en particulier dans l'oviducte (résultats non publiés). 


\section{LES SECRETIONS APOCRINES ET L'EPIDIDYME}

De même que la P25b bovine et la P26h de hamster qui sont GPI ancrées à la membrane plasmique du gamète mâle, d'autres protéines spermatiques acquises au cours du transit épididymaire se comportent comme des protéines membranaires intégrales lorsque soumises à différents types d'extraction [15]. Ceci suggère que ces protéines acquises par le spermatozoïde apparaissent dans le milieu intraluminal suite à une sécrétion de type inhabituel par les cellules épididymaires $[15,32]$. Yanagimachi en 1985, est le premier à démontrer que des vésicules membranaires s'associent à la surface du spermatozoïde lors de leur passage dans l'épididyme [53]. II émet alors l'hypothèse que ces vésicules modifieraient la composition en cholestérol membranaire des spermatozoïdes qui subissent des modifications majeures lors de la maturation épididymaire. La présence d'exosomes dans l'épididyme, nommés épididymosomes par certains, a été mise en évidence chez le rat [19], la souris [42], le hamster doré [34] et le taureau [24].

En plus des protéines ( $\mathrm{P} 26 \mathrm{~h}, \mathrm{P} 25 \mathrm{~b}$ et $\mathrm{P} 34 \mathrm{H})$ associées aux épididymosomes qui sont transférées aux spermatozoïdes au cours du transit épididymaire et qui leur confèrent la capacité de se lier à la zone pellucide, d'autres protéines associées aux exosomes d'origine épididymaire ont été identifiées. Chez la souris, la glutathion peroxydase de type 5 serait associée aux épididymosomes puis transférée aux spermatozoïdes afin de leur conférer une protection contre les dérivés actifs de l'oxygène [42]. L'ubiquitine est une autre protéine associée aux épididymosomes qui pourrait être impliquée dans l'élimination de spermatozoïdes anormaux dans l'épididyme $[14,20,31,51]$. Une cytokine, "Macrophage migration Inhibitory Factor" (MIF) serait également un produit de sécrétion apocrine de l'épididyme qui s'associe aux spermatozoïdes en cours de maturation [18, 21]. MIF s'associerait aux fibres denses des flagelles du spermatozoïde épididymaire et contrôlerait la formation de ponts disulfures dans ces structures flagellaires lors de la maturation post-testiculaire du gamète mâle [17].

Le mécanisme par lequel les protéines associées aux épididymosomes sont transférées aux spermatozoïdes lors de leur passage dans l'épididyme suscite de l'intérêt depuis peu. L'aldose réductase ainsi que la sorbitol déhydrogénase seraient également deux enzymes associées aux épididymosomes chez le bovin. Ces enzymes font partie du sentier métabolique des polyols et pourraient ainsi moduler la motilité des spermatozoïdes épididymaires [21, 22].

\section{L'INTERACTION ENTRE LES ÉPIDIDYMOSOMES ET LES SPERMATOZOÏDES}

L'étude des interactions entre les épididymosomes et les spermatozoïdes se produisant au cours de la maturation épididymaire nécessite de grandes quantités de matériel biologique. Afin d'élucider ces mécanismes, nous avons entrepris une série d'expériences en utilisant du matériel bovin provenant de l'abattoir. En utilisant cette espèce, il est possible de récupérer des quantités suffisantes de liquide intraluminal provenant de différentes régions épididymaires. Ainsi des préparations non contaminées de protéines solubles du liquide épididymaire, d'épididymosomes et de spermatozoïdes peuvent être obtenues à faible coût. Ce type de matériel ne peut être obtenu en utilisant les animaux de laboratoire et encore moins en utilisant du matériel biologique humain. Nous avons ainsi mis au point des protocoles expérimentaux permettant d'interroger les mécanismes d'interaction entre les épididymosomes et les spermatozoïdes épididymaires.

Des analyses électrophorétiques bidimensionnelles ont été effectuées sur les protéines provenant de liquide épididymaire. Celles-ci révèlent que la composition en protéines des épididymosomes diffère grandement des protéines solubles présentes dans le liquide épididymaire à partir duquel les épididymosomes ont été préparés. Une analyse comparative de ces profils démontre que les protéines associées aux épididymosomes contribuent peu à l'ensemble des protéines présentes dans le liquide épididymaire intraluminal. Ces différences permettent une analyse protéomique afin de mettre en lumière les particularités des épididymosomes et d'émettre des hypothèses concernant le rôle de ces microvésicules membranaires dans la maturation post-testiculaire des spermatozoïdes [21, 22].

En utilisant une approche plus mécanistique, nous avons biotinylé les protéines exposées à la surface d'épididymosomes purifiés afin d'étudier le mécanisme de transfert protéique vers les spermatozoïdes. Nous avons établi les conditions in vitro de transfert protéique de l'épididymosome vers le spermatozoïde. Ainsi, des épididymosomes préparés à partir du liquide intraluminal de la queue épididymaire sont caractérisés par un profil de protéines biotinylées complexe. L'association de ces protéines aux épididymosomes est très forte ; elle résiste à un traitement au Triton $X 100$ à $0,1 \%$. Suite à une incubation avec des spermatozoïdes de la tête épididymaire, seules quelques-unes de ces protéines associées aux épididymosomes sont transférées aux spermatozoïdes [23]. Ces protéines peuvent être localisées sur les spermatozoïdes en les révélant avec une avidine couplée à une peroxydase: l'avidine montre une très haute affinité pour la biotine. Or, les protéines transférées ne sont pas distribuées de façon uniforme à la surface du spermatozoïde et cette localisation peut varier d'une cellule à l'autre. Cette variabilité d'une cellule à l'autre réflète l'hétérogénéité des spermatozoïdes épididymaires. La région de l'acrosome et de la pièce intermédiaire du flagelle sont les sites privilégiés du transfert des protéines provenant des épididymosomes [23]. L'analyse densitométrique de "Western blots" des protéines transférées sur les spermatozoïdes permet de quantifier les protéines transférées des épididymosomes à un nombre prédéterminé de spermatozoïdes [21].

Le transfert des protéines des épididymosomes de la queue épididymaire vers les spermatozoïdes de la tête est sensible à la température, i.e. qu'il est plus efficace à $37^{\circ} \mathrm{C}$ qu'à des températures inférieures. Ceci laisse présumer que la fluidité membranaire des épididymosomes ou des 
spermatozoïdes pourrait être un facteur déterminant de l'efficacité du transfert. Ce transfert est saturable avec le temps, atteignant un plateau à environ 150 minutes de coincubation. Ce transfert protéique n'est donc pas un simple phénomène d'attachement non spécifique à la surface des spermatozoïdes. Le transfert protéique des épididymosomes vers les spermatozoïdes est également sensible au $\mathrm{pH}$ du milieu de co-incubation. Des co-incubations effectuées à des $\mathrm{pH}$ variant entre 5,5 et 8,0 montrent que le transfert protéique est optimal à $\mathrm{pH} 6,0-6,5$. II est intéressant de noter que ce $\mathrm{pH}$ correspond au $\mathrm{pH}$ intraluminal de l'épididyme [12, 36].

Un autre facteur déterminant du transfert protéique des épididymosomes vers le spermatozoïde est la présence du Zinc dans le milieu de co-incubation. En comparaison d'un milieu ne contenant pas de cation divalent, la présence de zinc favorise grandement la quantité de protéine transférée sur le spermatozoïde. Cet effet peut être annulé par l'ajout de chélateur de cations divalents tel EDTA. Ni le calcium et ni le magnésium n'ont cet effet bénéfique sur le transfert protéique. Considérant que l'épididyme est un tissu très riche en zinc [27], cette observation laisse croire que ce cation divalent joue un rôle important dans la maturation des spermatozoïdes lors du transit épididymaire [13]. II est à noter également que les prostasomes, les exosomes provenant des sécrétions prostatiques, sont également riches en zinc. La présence de zinc dans les épididymosomes reste par contre à être déterminée [43].

\section{CONCLUSION}

Le transfert de protéines associées aux épididymosomes vers les spermatozoïdes impliqué dans le processus de maturation post-testiculaire du gamète mâle est un phénomène hautement régulé. L'interaction entre ces vésicules sécrétées de façon apocrine par l'épithélium épididymaire et le spermatozoïde est probablement un élément clé dans l'acquisition du pouvoir fécondant du gamète mâle lors de son passage dans le tractus reproducteur mâle.

\section{Remerciements :}

Plusieurs étudiants gradués ont participé aux travaux de notre laboratoire qui sont décrits dans cette revue: Christian Gaudreault, Julie Girouard, Christine Guillemette, Carl Lessard et Sophie Parent. Trois stagiaires post-doctoraux ont également contribué à ces travaux, les Drs Franck Boué, Lucile Monfort et Fabrice Saez. Le Dr M.A. Fortier de I'Université Laval, le $\operatorname{Dr} M$ Nishibori de Université Okayama et le Dr S.K. Chung de l'Université de Hong Kong sont remerciés pour nous avons fourni différents anticorps indispensables à nos études. La collaboration du $\mathrm{Dr}$ $M$. Thabet de l'hôpital St-François dAssise (Québec, Qc) dans les études portant sur le matériel biologique humain est grandement appréciée. L'industrie de l'insémination artificielle du Canada, particulièrement "Boviteq Inc" (StHyacinthe, Qc) et le "Centre d'Insémination Artificielle du Québec" (St-Hyacinthe, Qc) sont remerciés pour nous avoir fourni des échantillons de semence bovine ainsi que des informations concernant les indices de fertilité. Ces travaux ont été financés par les "Instituts de Recherche en Santé du Canada", le "Conseil de Recherche en Sciences Naturelles et Génie" du Canada et le "Fond Québécois de Recherche sur la Nature et les Technologies".

\section{REFERENCES}

1. AGRAWAL Y., VANHA-PERTTULA T. : Effect of secretory particles in bovine seminal vesicle secretion on sperm motility and acrosome reaction. J. Reprod. Fertil., 1987, $79: 409-419$.

2. ARIENTI G., CARLINI E., DE COSMO A.M. et al. : Prostasome-like particles in stallion semen. Biol. Reprod., 1998, 59 : 309-313.

3. ARIENTI G., CARLINI E., POLCI A. et al. : Fatty acid pattern of human prostasome lipid. Arch. Biochem. Biophys., 1998, $358: 391-395$.

4. ARIENTI G., CARLINI E., SACCARDI C. et al. : CA. Role of human prostasomes in the activation of spermatozoa. J. Cell Mol. Med., 2004, 8 : 77-84.

5. AUMULLER G., WILHELM B., SEITZ J. : Apocrine secretionfact or artifact ? Anat. Anz., 1999,181: 437-446.

6. BERUBE B., LEFIEVRE L., COUTU L. et al. : Regulation of the epididymal synthesis of $\mathrm{P} 26 \mathrm{~h}$, a hamster sperm protein. J. Androl., 1996, $17:$ 104-110.

7. BERUBE B., SULLIVAN R. : Inhibition of in vivo fertilization by active immunization of male hamsters against a $26-\mathrm{kDa}$ sperm glycoprotein. Biol. Reprod, 1994, $51: 1255-1263$.

8. BOUE F., BERUBE B., DE LAMIRANDE E. et al. : Human sperm-zona pellucida interaction is inhibited by an antiserum against a hamster sperm protein. Biol. Reprod., 1994, 51 : 577-587.

9. BOUE F., BLAIS J., SULLIVAN R. : Surface localization of $\mathrm{P} 34 \mathrm{H}$ an epididymal protein, during maturation, capacitation, and acrosome reaction of human spermatozoa. Biol. Reprod., 1996, 54 : 1009-1017.

10. BOUE F., SULLIVAN R. : Cases of human infertility are associated with the absence of $\mathrm{P} 34 \mathrm{H}$ an epididymal sperm antigen. Biol. Reprod., 1996, 54 : 1018-1024.

11. BREITBART H., RUBINSTEIN S. : Characterization of Mg2+and $\mathrm{Ca2+-ATPase}$ activity in membrane vesicles from ejaculated ram seminal plasma. Arch. Androl., 1982, $9: 147-157$.

12. CAFLISCH C.R., DUBOSE T.D. JR. : Effect of vasectomy on in situ $\mathrm{pH}$ in rat testis and epididymis. Contraception, 1990, 42 : 589-595.

13. CALVIN H.I., YU C.C., BEDFORD J.M. : Effects of epididymal maturation, zinc (II) and copper (II) on the reactive sulfhydryl content of structural elements in rat spermatozoa. Exp. Cell Res., 1973, 81 : 333-341.

14. CHRISTOVA Y., JAMES P., MACKIE A. et al. : Molecular diffusion in sperm plasma membranes during epididymal maturation. Mol. Cell. Endocrínol., 2004, 216 : 41-46.

15. COOPER T.G. : Interactions between epididymal secretions and spermatozoa. J. Reprod. Fertil., 1998, Suppl 53 : 119-136.

16. DASSOULI A., DARNE C., FABRE S. et al. : Vas deferens epithelial cells in subculture: a model to study androgen regulation of gene expression. J. Mol. Endocrinol., 1995, $15: 129$ 141.

17. EICKHOFF R., BALDAUF C., KOYRO H.W. et al. : Influence of macrophage migration inhibitory factor (MIF) on the zinc 
content and redox state of protein-bound sulphydryl groups in rat sperm : indications for a new role of MIF in sperm maturation. Mol. Hum. Reprod., 2004, 10 : 605-611.

18. EICKHOFF R., WILHELM B., RENNEBERG H. et al. : Purification and characterization of macrophage migration inhibitory factor as a secretory protein from rat epididymis: evidences for alternative release and transfer to spermatozoa. Mol. Med., 2001, $7:$ :27-35.

19. FORNES M.W., BARBIERI A., CAVICCHIA J.C. : Morphological and enzymatic study of membrane-bound vesicles from the lumen of the rat epididymis. Andrologia, 1995, $27: 1-5$.

20. FRAILE B., MARTIN R., DE MIGUEL M.P. et al. : Light and electron microscopic immunohistochemical localization of protein gene product 9.5 and ubiquitin immunoreactivities in the human epididymis and vas deferens. Biol. Reprod., 1996, 55 : 291-297.

21. FRENETTE G., LESSARD C., MADORE E. et al. : Aldose reductase and macrophage migration inhibitory factor are associated with epididymosomes and spermatozoa in the bovine epididymis. Biol. Reprod., 2003, 69 : 1586-1592.

22. FRENETTE G., LESSARD C., SULLIVAN R. : Polyol pathway along the bovine epididymis. Mol. Reprod. Dev., 2004, 69 : 448-456.

23. FRENETTE G., LESSARD C., SULLIVAN R. : Selected proteins of "prostasome-like particles" from epididymal cauda fluid are transferred to epididymal caput spermatozoa in bull. Biol. Reprod., 2002, 67 : 308-313.

24. FRENETTE G., SULLIVAN R. : Prostasome-like particles are involved in the transfer of P25b from the bovine epididymal fluid to the sperm surface. Mol. Reprod. Dev., 2001, 59:115121.

25. GAUDREAULT C., LEGARE C., BERUBE B. et al. : Hamster sperm protein, $\mathrm{p} 26 \mathrm{~h}$ : a member of the short-chain dehydrogenase/reductase superfamily. Biol. Reprod., 1999, 61 : 264273.

26. GAUDREAULT C., MONTFORT L., SULLIVAN R. : Effect of immunization of hamsters against recombinant $P 26 \mathrm{~h}$ on fertility rates. Reproduction, 2002, $123: 307-313$.

27. GHOSH M., ROY S.K., CHOWDHURY A.R. : Zinc and copper content in rat epididymis and vas deferens. Endokrinologie, 1978, $71:$ 206-209.

28. GROOS S., WILHELM B., RENNEBERG H. et al. : Simultaneous apocrine and merocrine secretion in the rat coagulating gland. Cell Tissue Res., 1999, 295 : 495-504.

29. GUILLEMETTE C., THABET M., DOMPIERRE L. et al. : Some vasovasostomized men are characterized by low levels of P34H, an epididymal sperm protein. J. Androl., 1999, 20 : 214-219.

30. HERMO L., JACKS D. : Nature's ingenuity : bypassing the classical secretory route via apocrine secretion. Mol. Reprod. Dev., 2002, 63 : 394-410.

31. JONES R. : Sperm survival versus degradation in the Mammalian epididymis : a hypothesis. Biol. Reprod., 2004, 71 : 1405-1411.

32. KIRCHHOFF C., HALE G. : Cell-to-cell transfer of glycosylphosphatidylinositol-anchored membrane proteins during sperm maturation. Mol. Hum. Reprod., 1996, 2 : 177-184.

33. KRAVETS F.G., LEE J., SINGH B. et al. : Prostasomes : current concepts. Prostate, 43, $2000: 169-174$.

34. LEGARE C., BERUBE B., BOUE F. et al. : Hamster sperm antigen P26h is a phosphatidylinositol-anchored protein. Mol. Reprod. Dev., 1999, 52 : 225-233.

35. LEGARE C., GAUDREAULT C., ST-JACQUES $S$. et al. : $\mathrm{P} 34 \mathrm{H}$ sperm protein is preferentially expressed by the human corpus epididymidis. Endocrinology, 1999, $140: 3318-3327$.

36. LEVINE N., KELLY H. : Measurement of $\mathrm{pH}$ in the rat epididymis in vivo. J. Reprod. Fertil., 1978, 52 : 333-335.

37. MANIN M., LECHER P., MARTINEZ A., et al. : Exportation of mouse vas deferens protein, a protein without a signal peptide, from mouse vas deferens epithelium : a model of apocrine secretion. Biol. Reprod., 1995, 52 : 50-62.

38. MINELLI A., MORONI M., MARTINEZ E. et al. : Occurrence of prostasome-like membrane vesicles in equine seminal plasma. J. Reprod. Fertil., 1998, $114:$ 237-243.

39. MONTFORT L., FRENETTE G., SULLIVAN R. : Sperm-zona pellucida interaction involves a carbonyl reductase activity in the hamster. Mol. Reprod. Dev., 2002, 61 : 113-119.

40. NICKEL W. : The mystery of nonclassical protein secretion. A current view on cargo proteins and potential export routes. Eur. J. Biochem., 2003, $270: 2109-2119$.

41. PARENT S.L.L., BRINDLE Y., SULLIVAN R. : Bull subfertility is associated with low levels of a sperm membrane antigen. Mol. Reprod. Dev., 1999, 52 : 57-65.

42. REJRAJI H., VERNET P., DREVET J.R. : GPX5 is present in the mouse caput and cauda epididymidis lumen at three different locations. Mol. Reprod. Dev., 2002, 63 : 96-103.

43. RONQUIST G. : Zinc enrichment in prostasomes. Int. J. Androl., 1998, 21 : 233-234.

44. RONQUIST G., BRODY I., GOTTFRIES A. et al. : An Mg2+ and $\mathrm{Ca} 2+$-stimulated adenosine triphosphatase in human prostatic fluid-part II. Andrologia, 1978, $10: 427-433$.

45. RONQUIST G., NILSSON B.O. : The Janus-faced nature of prostasomes : their pluripotency favours the normal reproductive process and malignant prostate growth. Prostate Cancer Prostatic Dis., 2004, $7: 21-31$.

46. SAEZ F., FRENETTE G., SULLIVAN R. : Epididymosomes and prostasomes : their roles in posttesticular maturation of the sperm cells. J. Androl., 2003, $24: 149-154$.

47. SULLIVAN R. : Male fertility markers, myth or reality. Anim. Reprod. Sci., 2004, 82-8: 341-347.

48. SULLIVAN R., BLEAU G. : Interaction between isolated components from mammalian sperm and egg. Gamete Res., 1985, 12 : 101-116.

49. SULLIVAN R., FRENETTE G., LESSARD C. et al. : Sperm antigen acquisition in the epididymis : A role for epididymosomes. In : Hinton B.T., Turner T. eds. Epididymis III. Charlottesville (VA, USA), Van Doren Company, 2003, 130-136.

50. SULLIVAN R., ROBITAILLE G. : Heterogeneity of epididymal spermatozoa of the hamster. Gamete Res., 1989, 24 : 229236.

51. SUTOVSKY P., MORENO R., RAMALHO-SANTOS J. et al. : A putative, ubiquitin-dependent mechanism for the recognition and elimination of defective spermatozoa in the mammalian epididymis. J. Cell Sci., 2001, $114: 1665-1675$.

52. UTLEG A.G., YI E.C., XIE T. et al. : Proteomic analysis of human prostasomes. Prostate, 2003, 56 : 150-161.

53. YANAGIMACHI R., KAMIGUCHI Y., MIKAMO K. et al. : Maturation of spermatozoa in the epididymis of the Chinese hamster. Am. J. Anat., 1985, $172: 317-330$. 
Communication au $X X I^{\circ}$ Congrès de la Société d'Andrologie de Langue Française, Clermont-Ferrand, 9-11 Décembre 2004.

Manuscrit reçu : décembre 2004 ; accepté décembre 2004

\section{ABSTRACT}

Apocrine secretion in accessory sex glands

Robert SULLIVAN, Gilles FRENETTE, Christine LEGARE

Although differentiated spermatozoa are incapable of transcriptional or translational activity, the macromolecule composition of their surface undergoes major changes during passage in the male reproductive tract. These changes are due to sequential, well orchestrated interactions between male reproductive tract secretions and the spermatozoan, particularly as it travels along the epididymis. Some of the sperm proteins acquired during maturation along the excurrent duct behave as integral membrane proteins. In fact, some epididymis-derived proteins are glycosyl phosphatidylinositol (GPI) anchored to the sperm plasma membrane, which raises the question of the mechanisms by which these proteins are secreted by the epididymal epithelium.

Our laboratory has identified a family of proteins added to the sperm surface during epididymal transit. These proteins are GPI anchored and the deduced amino acid sequences revealed the absence of a signal peptide in the $\mathrm{N}$-terminal sequence of these proteins. These findings suggest that these proteins are secreted in an apocrine manner by the epididymal epithelium. Apocrine secretion involves formation of a cytoplasmic bleb in the apical region of the epithelial cells that is released into the intraluminal compartment. These blebs disintegrate and release small membranous vesicles generically called exosomes or epididymosomes when secreted by the epididymis. These vesicles are rich in sphingomyelin and are characterized by a high cholesterol/phospholipid ratio. Exosomes have also been reported to be secreted by the prostate, epididymis, and vas deferens.

Many proteins are associated with epididymosomes, and some of them are selectively transferred to spermatozoa during epididymal transit. We have identified some of these proteins by a proteomic approach, including an aldose reductase and a sorbitol dehydrogenase, two enzymes involved in the polyol pathway. Another protein associated with epididymosomes that is transferred to spermatozoa is a cytokine called "Macrophage migration Inhibitory Factor". During epididymal maturation, this protein is associated with the outer dense fibers of the sperm flagellum and modulates the formation of disulfide bonds. It is hypothesized that both the polyol pathway and apocrine secretion of MIF by the epididymal epithelium modulate sperm motility during transit along the male reproductive tract. Further research is needed to understand the functions of other proteins secreted in an apocrine manner by the various glands of the male reproductive tract.

Key words : epididymis, exosomes, maturation, secretion, proteins 